\title{
Reseñas
}

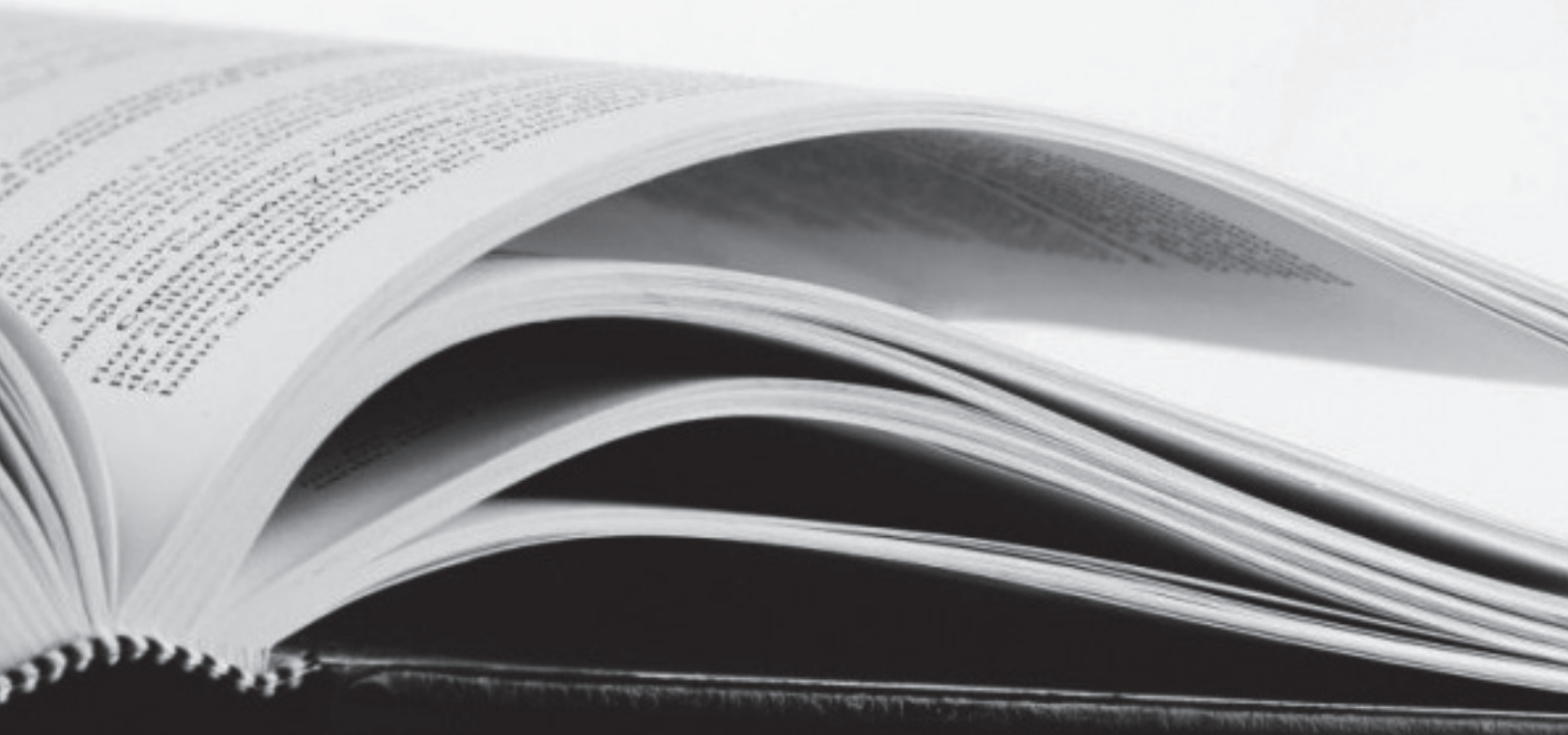



Universidad de Guadalajara

DERECHO GLOBAL. ESTUDIOS SOBRE DERECHO Y JUSTICIA

Año 2022, Vol. VII. Número 20, Marzo - Junio, ISSN: 2448-5128 e-ISSN: 2448-5136

https://DOI.org/ 10.32870/dgedj.v7i20.487

KENNIER JOSÉ GARAY HERAZO

Universidad de Antioquia, Colombia

Kennier.garay@udea.edu.co

\section{CONSENTIMIENTO INFORMADO Y Y Responsabilidad Médica}

\section{INFORMED CONSENT AND MEDICAL LIABILITY}

Cómo citar el artículo:

Garay K, (2022). Reseña: Consentimiento Informado y Responsabilidad Médica. Derecho Global, Estudios sobre Derecho y Justicia, VII (20) Falta la paginación https://DOI.org/ 10.32870/dgedj. v7i20.487 pp. $263-267$

Recibido: 25/05/21 Aceptado: 22/11/21 
A finales de 2019 se conocieron las primeras noticias sobre una epidemia que afectó inicialmente a China, pero que al cabo de unos meses se desplegó por otros lugares del mundo hasta alcanzar la categoría de pandemia. Su nombre se volvió tan común que desde entonces hace presencia en casi todos los espacios de la actual cotidianidad. Se alude, por supuesto, a la denominada COVID-19 o enfermedad por el nuevo coronavirus SARS-CoV-2. Los impactos de esta enfermedad han sido muy diversos, pues deambulan sin tregua y a ritmos cambiantes por cada uno de los lugares que la padecen. En algunos ha dado pie, además, a crisis o situaciones de emergencia social y económica de alcance general o por sectores socioeconómicos determinados. Problemáticas que surgieron, en algunos casos, genuinamente con la pandemia; en otros ya preexistían y, a lo sumo, se agudizaron.

Por otro lado, existe una amplia gama estrategias, respuestas, medidas y acciones que han sido llevadas a cabo por los Estados, Gobiernos, autoridades públicas y particulares frente a cada una de estas situaciones. Generalmente, estas respuestas se han manifestado como marcos regulatorios, normativa, o como la ejecución misma de políticas públicas con las que se espera gestionar la pandemia. Medidas necesarias y pertinentes, argumentan algunos con razonamientos de tipo epidemiológico y de salud pública. Excesivas, restrictivas y poco eficaces para garantizar otros derechos humanos y fundamentales que se ven afectados por causa de la pandemia, sostienen otros desde posiciones más filosóficas o libertarias. Sin embargo, debido a su 'naturaleza' conflictiva, urgencia y novedad, se necesitarán más que los simples debates jurídico-políticos de proporcionalidad, ponderación o razonabilidad para la toma de decisiones administrativas y judiciales que sean requeridas para su adecuada gestión.

Quizás el principal entorno para evidenciar este tipo de conflictos o tensiones de derechos sea el médico-sanitario. Su implicación de amenaza directa frente a derechos como la vida y la salud se torna problemática por la novedad y falta de resultados de investigaciones científicas concluyentes; la falta de personal médico-sanitario especializado; la inadecuada infraestructura y deficiencias técnicas en algunas instalaciones y equipos médicos; las

\section{DERECHO GLOBAL. ESTUDIOS SOBRE DERECHO Y JUSTICIA}


precarias condiciones laborales y de contratación para el personal médico-sanitario; las deficiencias en materia de cobertura; los vacíos regulatorios e interpretaciones lesivas en materia de riesgos laborales y dotación de bioseguridad; la restricción de algunos servicios de asistencia, promoción y prevención de la salud, diagnósticos e intervenciones y procedimientos quirúrgicos; la abstención frente al uso de otros servicios por temor o prevención frente al contagio, entre otras situaciones.

Circunstancias que afectan al personal médico-sanitario, entidades prestadoras y promotoras de salud, administradoras de riesgos laborales y, especialmente, a pacientes. Estos últimos podrían ver afectados sus derechos a la vida, salud e integridad física y psíquica, de muchas formas en el contexto de la actual pandemia. Sin embargo, los derechos referidos se encuentran en relación directa con otros derechos igualmente importantes, pero usualmente relegados a los que no se les brinda tanta atención, como ocurre - por ejemplo- con el derecho al consentimiento informado.

Es muy probable que en estos momentos se tramiten denuncias, quejas, indagaciones preliminares, investigaciones y procesos administrativos o judiciales, cuyo objeto esté relacionado con casos de responsabilidad médica por probable desconocimiento del derecho al consentimiento informado durante la pandemia. Otra razón más para dedicar estas líneas al tema en cuestión y reflexionar sobre sus implicaciones jurídicas; tanto para los pacientes como para el personal y entidades médico-sanitarias.

A finales de 2017, el abogado Juan Guillermo Agón López publica el libro titulado Consentimiento informado y responsabilidad médica, bajo el respaldo del sello editorial internacional La Ley-Wolters Kluwer. Texto que es el resultado de sus investigaciones durante su proceso de formación doctoral en Derecho Privado en la Universidad de Salamanca, España. Aunque el lector debe tener en cuenta que este libro ofrece una visión mucho más amplia y completa que la de la lectura coyuntural propuesta, el texto podría ser de utilidad para pacientes; personal médicosanitario; investigadores; estudiantes; abogados litigantes o asesores; autoridades administrativas o judiciales, en situaciones y casos en los que se llegaren a suscitar 
discusiones por posibles vulneraciones al derecho al consentimiento informado, a la vida o a la salud en medio de intervenciones médicas durante el periodo de pandemia. Así las cosas, el libro puede brindar insumos para la construcción de las respuestas a interrogantes como los que, a manera de ejemplo, se plantean a continuación: ¿qué pasa en aquellos casos en los que no se haya respetado o garantizado el derecho al consentimiento informado a los pacientes sometidos a intervenciones médicas durante la pandemia? ¿cómo entender dicho derecho en general y para situaciones como la actual? ¿existen razones válidas para sacrificar este derecho en favor de otros derechos? ¿qué criterios deben tenerse en cuenta para evaluar la responsabilidad del médico o de la entidad de salud cuando no se garantice el derecho al consentimiento informado del paciente?

En cuanto a su estructura, el libro inicia con el prólogo a cargo del catedrático y experto español en Derecho Civil, Eugenio Llamas Pombo, quien realiza una presentación general y panorámica de la materia abordada. Posteriormente, el autor desarrolla sus contenidos temáticos en tres capítulos. El primer capítulo denominado el consentimiento informado y el derecho a la información del paciente: consideraciones generales. El segundo capítulo se titula el régimen jurídico del consentimiento informado. Por último, está el tercer capítulo, cuyo título es la responsabilidad médica por el incumplimiento de los deberes de información y de consentimiento informado.

Frente al primer capítulo se resalta la valiosa labor de conceptualización lograda por el autor frente al consentimiento informado como derecho del paciente. Para esto se lleva a cabo un interesante ejercicio genealógico que se vale de herramientas propias de la historia, en conjunción con aspectos de la bioética y el derecho comparado, que le permiten presentar y analizar sus fundamentos y 'naturaleza' jurídica. En especial, es bastante ilustrativo el recorrido que al respecto se hace de forma sucinta por los antecedentes de este tema en Estados Unidos, Reino Unido, Francia, Alemania e Italia. Así como frente a sus primeras regulaciones internacionales europeas y española.

\section{DERECHO GLOBAL. ESTUDIOS SOBRE DERECHO Y JUSTICIA}


En el segundo capítulo, propiamente jurídico, se lleva a cabo una completa presentación de tipo deóntico sobre los elementos que conforman el derecho al consentimiento informado. En ella se indaga sobre la titularidad del derecho; así como sobre los deberes predicables en cabeza de los sujetos obligados; el objeto y contenido de la información; la forma que debe revestir la información y el consentimiento en sí; las obligaciones y cargas probatorias con respecto a información y el consentimiento, así como su revisión en el ámbito temporal. También se revisa la figura de la representación jurídica y sus implicaciones con ocasión de este derecho.

En atención a la perspectiva de utilidad sugerida para la lectura ante el contexto de la pandemia por COVID-19, debe mirarse con especial cuidado los acápites que en el segundo capítulo se dedican a estudiar las excepciones al consentimiento informado; ya sea porque: (i) existe un riesgo para la salud pública, o (ii) existe un riesgo inmediato para la integridad física o psíquica del paciente y no es posible obtener su autorización antes de la respectiva intervención.

Por último, el tercer capítulo presenta y evalúa las posibles consecuencias jurídicas que podrían surgir de la afectación al derecho al consentimiento informado, a la integridad física y psíquica, desde una mirada guiada por el régimen de responsabilidad civil. Para lograrlo, el autor se detiene a revisar los principales presupuestos que configurarían la responsabilidad de dicho tipo, en este caso: la relación jurídica que se da entre el personal médico-sanitario y el paciente; la denominada culpa médica; la relación de causalidad y la imputación objetiva; así como los posibles daños generados y eventualmente susceptibles de indemnización. Sin duda alguna, en estas breves líneas se escapan muchas perspectivas de lectura y análisis que podría encontrar y realizar cada uno de los lectores del texto reseñado. Máxime, si se tiene en cuenta que la actual pandemia aún está en curso y, en consecuencia, su comprensión, implicaciones e historia todavía son incompletas y quizás requeriremos mucho más tiempo para ello. 


\section{BIBLIOGRAFÍA}

Libro: $\quad$ Consentimiento informado y responsabilidad médica

Autor: Juan Guillermo Agón López

Editorial: La Ley-Wolters Kluwer

Lugar: Madrid, España

Año: 2017

Páginas:

$371 \mathrm{pp}$. 